\title{
Cutting velocity accuracy as a criterion for comparing robot trajectories and manual movements for medical industry
}

\author{
A.A. Vorotnikov ${ }^{1}{ }^{*}$, D.D. Klimov ${ }^{1}$, E.V. Romash ${ }^{1}$, O.S. Bashevskaya ${ }^{1}$, Yu. V. Poduraev ${ }^{1}$, E.A. Bazykyan ${ }^{2}$, and \\ A.A. Chunihin ${ }^{2}$ \\ ${ }^{1}$ Moscow State University of Technology "STANKIN", 1 Vadkovsky per., Moscow 127055, Russian Federation \\ 2 Moscow State University of Medicine and Dentistry named after A.I. Evdokimov, 20/1 Delegatskaya ul., Moscow 127473, \\ Russian Federation
}

Received: 1 September 2017 / Accepted: 1 December 2017

\begin{abstract}
Industrial robots perform technological operations, such as spot and arc welding, machining and laser cutting along different trajectories within their performance characteristics. The evaluation of these characteristics is carried out according to the criteria of the standard ISO 9283. The criteria of this standard are applicable in industrial manufacturing, but not in the medical industry, as they are not developed in the framework of medical tasks. Therefore, it is necessary to evaluate according to criteria built on different principles. In this article, the question of comparative evaluation of trajectories from program movements of a robot and manual movements of a surgeon, arising during the development of robotic medical complexes using industrial robots, is considered. A comparative evaluation is required to prove the expediency of automating medical operations in maxillofacial surgery. This study focuses on the estimation of velocity accuracy of a medical instrument. To obtain the velocity of the medical instrument, coordinates of the trajectory points from the program movements of the robot KUKA LWR4+ and trajectories from the manual movements of a professional surgeon have been measured. The measurement was carried out using a coordinate measuring machine, the laser tracker Leica LTD800. The accuracy estimation was carried out by two criteria: the criterion set out in the ISO 9283 standard, and the developed alternative criterion, the description of which is presented in this article. A quantitative comparative evaluation of the trajectories of a robot and a surgeon was obtained.
\end{abstract}

Keywords: Medical robotics / laser tracker / trajectory evaluation / accuracy / diode laser

\section{Introduction}

At present, during medical operations in maxillofacial surgery, a diode laser with a pulsed pumping driver [1-4] is often used for cutting. It is because the use of diode lasers [5-10] improves treatment of patients. It reduces the trauma of surgical intervention, provides reliable hemostasis, creates sterility in the wound, reduces pain and swelling in the postoperative period, shortens the regeneration period of the tissues and forms soft elastic scars in comparison with excision with a scalpel. The diode laser is used to perform various operations [11-13]. In [14], researchers developed a nanosecond diode laser, which allows the removal of epulides. A low-level diode laser with low-energy density range can influence on bone tissue with biostimulatory effects on bone tissue prone to various pathologies [15]. Analysis of the mechanical properties of bones susceptible to osteoporosis is described

\footnotetext{
* e-mail: aavorotnikov90@gmail.com
}

in [16]. Such medical operations should be carried out under appropriate environmental conditions, as detailed in [17].

Recently, robotic medical systems are being actively developed, designed to partially or completely perform certain medical tasks, helping the surgeon in operating [1820]. For example, the researchers [21] are working on the creation of a robotic system STAR, capable of autonomously performing the sewing of incisions on soft tissues. The positioning system is a lightweight robot with 7 degrees of freedom (KUKA LWR4+). The robotic arm is kinematically similar to the human hand, which makes it a good candidate for use in robotic-assisted surgery. The article [22] describes the robotic system RONNA, which consists of two industrial robots, with a total of 13 degrees of freedom. The strong and rigid KUKA KR6 is used for targeting of planned operating points and the smaller. KUKA LWR4+ is used as an assistant. It is also suggested to apply industrial robots in neurosurgery in the brain area [23]. However, at the moment, studies with the approach of using industrial robots in laser maxillofacial surgery are extremely rare. 
With manual movements of the tip of the diode laser, the surgeon can perform tissue cuts depending on certain values of laser radiation power, pulse time and time between pulses. These parameters are cutting modes from the surgeon. It is possible to automate the movements of the tip of the diode laser, shifting the function of moving the medical instrument to the robot [24-26]. To apply the robot to perform operations [14-17], it is necessary to quantify its advantages in comparison with the surgeon. Metrological [27-29] comparison of the movements of the medical instrument is expedient to carry out according to trajectories predetermined for both the robot and the surgeon, which are a means of obtaining quantitative information about the movement. Typical trajectories are defined by analysis of typical medical operations in maxillofacial surgery. Using the robot, there is an expansion of the surgeon's cutting modes due to the additional ability to control and adjust the velocity of movement of the medical instrument. The set of obtained data on the velocity of the medical instrument on typical trajectories allows formulating one of the criteria according to which it is possible to compare the program movements of the robot and the manual movements of the surgeon.

This article presents the results of experimental studies comparing the velocity of manual and program movements of a surgeon and a robot, as well as a quantitative evaluation of their comparison by two criteria: cutting velocity accuracy, presented in the standard ISO 9283 [30], and the criterion developed by authors of this article, the arithmetic mean of the cutting velocity accuracy. The developed criterion differs significantly from the one presented in the standard; it includes an accounting of the human factor.

\section{Methods}

\subsection{Experimental setup}

The experimental setup includes two parts. The first part: for measurement of the coordinates of the points of the trajectories made during the robot's program movements and the second part: for measurement of the coordinates of the points of the trajectories made during the surgeon's manual movements. First and second parts of the experimental setup are shown in Figures 1 and 2, respectively.

The first part, an experimental setup for measuring trajectories from program movements performed by the robot, includes the following set of equipment:

- laser coordinate measuring machine, the laser tracker (1) LTD 800;

- medical robotic manipulator (2) KUKA LWR4+;

- robot work tool (3).

The work tool of the robot consists of a mechanical interface (4) connecting the holder of the optical waveguide (5) to the flange of the robot (6). To be able to measure the coordinates of the trajectory points, a reflector (7) of the laser tracker is installed on the mechanical interface.

The second part of the experimental setup for measuring trajectories from manual movements performed by a surgeon includes:

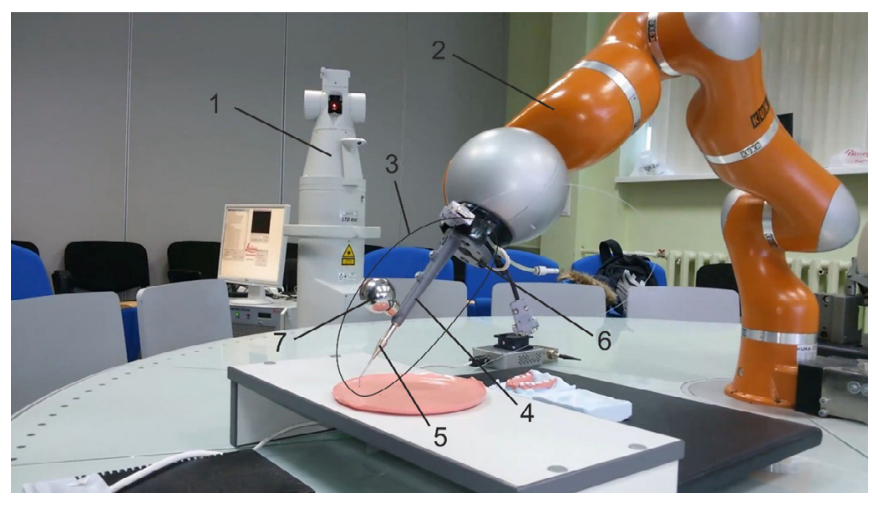

Fig. 1. First part: experimental setup for measuring trajectories from program movements of the robot.

- laser coordinate measuring machine, the laser tracker (1) LTD 800;

- robot work tool (8).

One of the main requirements for medical personnel to the design of a work tool for measuring manual movements was ergonomics since the manual movements performed by a surgeon should not be limited by the excess weight and dimensions of metrological devices to obtain reliable trajectories. In this regard, for the measurement of the coordinates of the points connected with the holder of the optical waveguide (5), it is necessary to use only a laser tracker. This CMM allows you to measure the coordinates of the reflector (9) having a weight not exceeding $7 \mathrm{~g}$ (the reflector (7) weights $170 \mathrm{~g}$ ). Also, the reflector is mounted on a magnetic base (10) weighting about $3 \mathrm{~g}$. Accordingly, the total weight of the measuring device that is to be connected to the optical waveguide holder is about $10 \mathrm{~g}$, which provides ergonomics for manual movements regarding surgery. The working tool of a surgeon for measuring trajectories from manual movements is shown in Figure 2.

The experimental setup allows measuring the Cartesian coordinates associated with the holder of the optical waveguide, both during manual movements of the surgeon and during program movements of the robot. The set of measured Cartesian coordinates constitutes the trajectory of motion of the optical waveguide holder. The advantage in ergonomics from surgery leads to a lack of the ability to measure the orientation of the work tool. As such, there is no need to measure orientation. The tolerance for the deviation of the angular position of the tool tip during the operation of both the robot and the surgeon is $\pm 5^{\circ}$.

After the analysis of standard operations in maxillofacial surgery, a set of required typical trajectories is formed, along which the robot and surgeon's movements will be carried out. Typical trajectories are linear $l_{i}\left(x_{i}, y_{i}, z_{i}\right)(11)$, semilunar $h_{i}\left(x_{i}, y_{i}, z_{i}\right)(12)$ and scalloped $f_{i}\left(x_{i}, y_{i}, z_{i}\right)(13)$ trajectories ( $i-$ number of measured points coordinates), which are shown in Figure 2. During the experiments, a manual movement is carried out by an experienced surgeon, programmed movements are also assigned by a surgeon, but using the robot KUKA LWR4+ via an intelligent interaction system. 


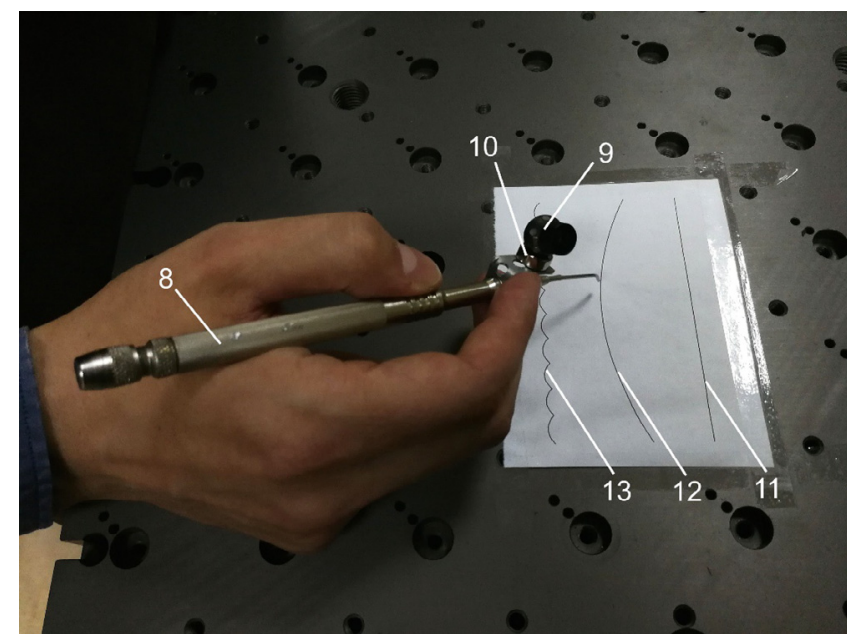

Fig. 2. Second part: experimental setup for measuring trajectories from manual movements of the surgeon.

Obtaining coordinate values of points of which the trajectory consists is performed using continuous scanning of the reflector position during its movement with the frequency of $300 \mathrm{~Hz}$.

In carrying out experiments to compare the movements of the robot and the manual movements of the surgeon, instead of the biological tissue, the surface of the table (14) with minimal flatness is used to estimate the manual movements. During determining the movements of the robot, the table surface is set using the robot's program. This is necessary to exclude the errors from the result of the evaluation associated with the difficulties of setting trajectories and comparing them on surfaces with a complex form of a biological tissue.

\subsection{Cutting velocity accuracy as a criterion for comparing program and manual movements}

To cut the required depth and width, it is necessary to constantly maintain the nominal (set) cutting velocity or, in other words, the velocity of the tip of the medical instrument $V_{n}$ (cutting velocity) at each point of the desired trajectory. If this requirement is not observed at a low velocity, the patient can receive additional microtrauma, burns due to an excess of laser radiation at the moment of cutting during the operation. At a high velocity, it is possible to undercut, so in this situation, it will be necessary to make an additional pass along the same trajectory, which in case of manual cutting will also cause additional injuries because a person cannot exactly repeat the passage along the same trajectory.

One of the possible criteria for comparing the movements of the robot and the manual movements of the surgeon is the cutting velocity accuracy of the medical instrument $\delta_{V}$. This criterion is presented in the standard ISO $9283: 1998$ [30] as a criterion evaluating the quality of the robot. This article considers the possibility of its application for assessing the quality of manual movements of the surgeon. Therefore, the determination of the cutting velocity accuracy $\delta_{V}$ both the robot and the surgeon is carried out by this [9] standard using the following formula:

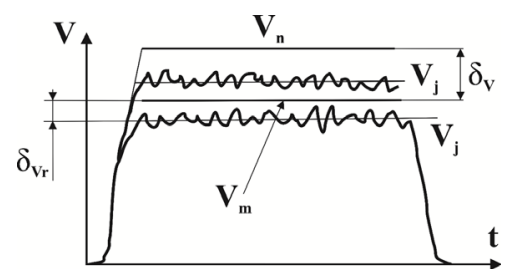

Fig. 3. Criteria for comparing manual movements and the robot movements $\delta_{V}$ and $\delta_{V r}$.

$$
\delta_{V}=\frac{\left|V_{m}-V_{n}\right|}{V_{n}} \cdot 100 \%,
$$

where $V_{n}$ - predetermined cutting velocity, $V_{m}-$ the average cutting velocity, is defined as the arithmetic mean of the cutting velocities from each trajectory pass $V_{j}$ ( $j=1 \ldots Q$, where $Q-$ number of passes). In turn, $V_{j}-$ the cutting velocity of one pass is defined as the arithmetic mean of the values of the current cutting velocity $V_{t k}$ on the trajectory. The arithmetic mean of the cutting velocity accuracy $\delta_{V}$, nominal cutting velocity $V_{n}$, average cutting velocity $V_{m}$ and the average cutting velocity at one pass along the trajectory $V_{j}$ are shown in Figure 3.

The current value of the cutting velocity $V_{t k}$ in each $k$ segment of any typical trajectory is calculated in accordance with the following formula:

$$
V_{t k}=\frac{r_{t k}}{T}
$$

Since the coordinates of the points were taken at a frequency of $v=300 \mathrm{~Hz}$, the time through which each new coordinate $T$ is obtained as $0,003(3) \mathrm{s}$. The distance $r_{t k}$ between each $i$ point and $(i+1)$ point of any typical trajectory $\left(q_{i}\left(x_{i}, y_{i}, z_{i}\right)-l_{i}, h_{i}\right.$ or $\left.f_{i}\right)$ is defined as follows:

$$
r_{t k}=\sqrt{\left(x_{i+1}-x_{i}\right)^{2}+\left(y_{i+1}-y_{i}\right)^{2}+\left(z_{i+1}-z_{i}\right)^{2}},
$$

where $\left(x_{i}, y_{i}, z_{i}\right)$ coordinates of any typical trajectory of both robot and surgeon. $i$ - the number of measured coordinates of points, $k$, the number that defines the segment $r_{t k}$ for the current velocity. With the maximum number of points $i=M$, the maximum number of measurements of the current velocity $k=M-1$.

\subsection{Features of setting the robot's trajectories}

Before presenting data on the experiments, it should be noted that when performing experiments to pass the scalloped trajectory with the help of the robot, there is a feature associated with setting this trajectory. When testing the scalloped trajectory, the robot can pass the reference points with or without smoothing. In the case of passage without smoothing, the robot must stop at the start and end point of each semicircle of the entire scalloped trajectory. Due to the lack of smoothing, the current cutting velocity $V_{t k}$ is reduced to zero when approaching the start or end point of each semicircle, which affects the total time of the trajectory. In the case of passage along a 


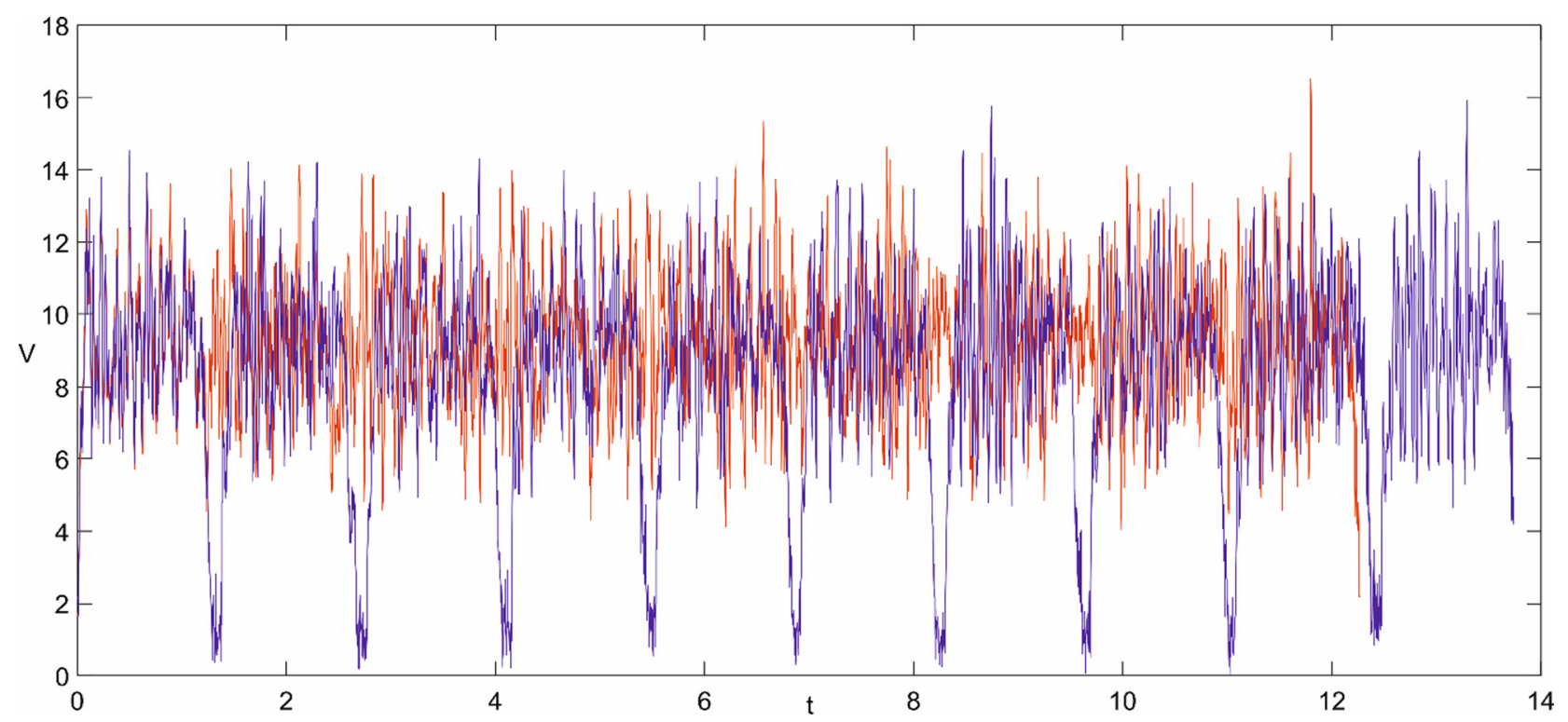

Fig. 4. The change of current cutting velocity $V_{t k}(\mathrm{~mm} / \mathrm{s})$ from the measurement time $t_{t k}(\mathrm{~s})$ of the coordinates of the position of the medical instrument on robot trajectory with smoothing (color, red) and without smoothing (color, blue).

scalloped trajectory with smoothing, an accuracy loss occurs at the approach to the start or end point of each semicircle, but the movement velocity remains. For the experiments conducted, the maximum loss in accuracy is $0.18 \mathrm{~mm}$, which is not so critical for most cuts and will allow maintaining the required velocity throughout the entire trajectory. In the case when this loss of accuracy is critical, it is possible to correct the trajectory as long as this value is measured. Figure 4 shows the graphs of the change in the current cutting velocity $V_{t k}$ from the time of the measurement $t_{t k}$ of the position coordinates of the medical instrument when the robot moves along the scalloped trajectory with and without smoothing. This feature is typical only for robots with a closed control system. If there is an open control system, you can set the path without smoothing at a constant velocity.

\section{Results and discussion}

\subsection{Preliminary experiments for the analysis of trajectories from manual movements}

Before comparing the surgeon and the robot we also conducted preliminary experiments necessary for understanding the quality of a surgeon's cutting to obtain reliable data about the resulting cutting velocity. There is a big difference in trajectories obtained from a trained surgeon and a surgeon who has not been trained in laser surgery. Therefore, it is advisable to show this difference. Typical trajectories carried out by surgeons are divided into two categories: trajectories, the passage through which was carried out in the presence of a support point, i.e., the surgeon's hand touches the surface of the table during the passage, and the trajectory without touching ("in the air"). This is due to the need for the surgeon to hold the cutting tool differently during operations, depending on the openness of the operating field.
In carrying out preliminary experiments, for comparison of both surgeons and the robot, it was required to maintain a nominal cutting velocity equal to $10 \mathrm{~mm} / \mathrm{s}$ at each distance $r_{t k}$ for any typical trajectory, the obtained trajectory data are shown in Figure 5. To compare the velocity of the tip of a medical instrument within a single sample trajectory, both the robot and the surgeons pass the same path. The values of the current velocity $V_{t k}$ are obtained at one pass on all typical trajectories. When passing through each trajectory, approximately 2500 measurements of the current velocity were obtained by scanning. Scalloped trajectories are set to the robot with smoothing. Comparative graphs illustrating the value of the current cutting velocity $V_{t k}$ from the time of the coordinate measurement $t_{i}$ at one pass for each typical trajectory by the robot and the untrained surgeon are shown in Figure 5. The graphs of the value change of current cutting velocity $V_{t k}(\mathrm{~mm} / \mathrm{s})$ from the measurement time $t_{t k}(\mathrm{~s})$ of the coordinates of the medical instrument position for manual movements of the untrained surgeon with a support point are (color, blue), without support point (color, green) and when moving with the help of the robot (color, red) along the typical trajectories (a - linear, b - semilunar, c - scalloped). When examining these graphs, a different time of completion of measurements $t_{i}$ on each typical trajectory was revealed with manual movements of an untrained surgeon with both a support point and without one. This is due to the excess of the current cutting velocity $V_{t k}$ relative to the nominal $V_{m}$ for all manual movements except for the scalloped trajectory with the support point. On the given trajectory there is a reverse: the value of the nominal cutting velocity $V_{m}$ exceeds the values of the current cutting velocity $V_{t k}$. The graphs also show the different stop times of the robot. This is because, regardless of the fact that the nominal path between the first and last point of each sample trajectory during the experiment is $100 \mathrm{~mm}$, due to the bends of the 

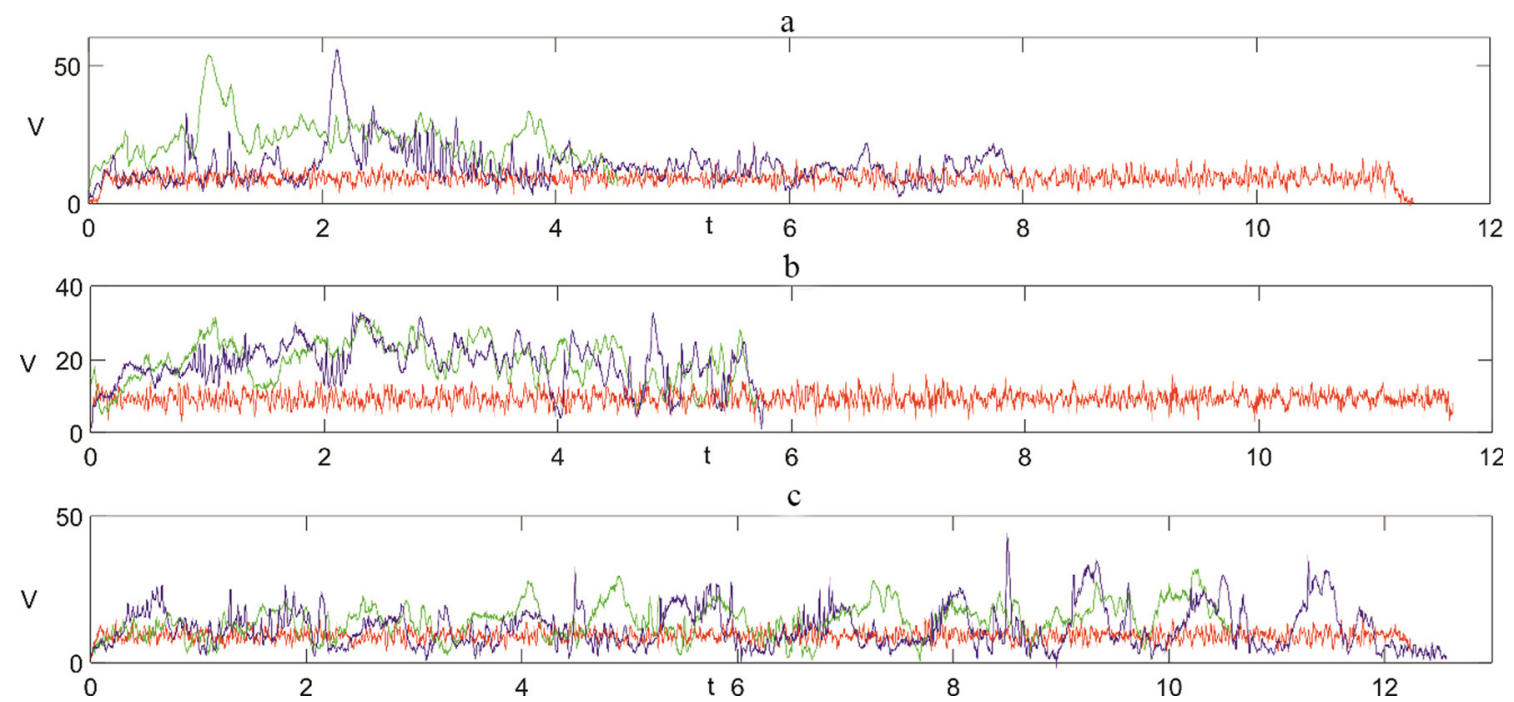

Fig. 5. Graphs of the value change of current cutting velocity $V_{t k}(\mathrm{~mm} / \mathrm{s})$ from the measurement time $t_{t k}(\mathrm{~s})$ of the coordinates of the position of the medical instrument.
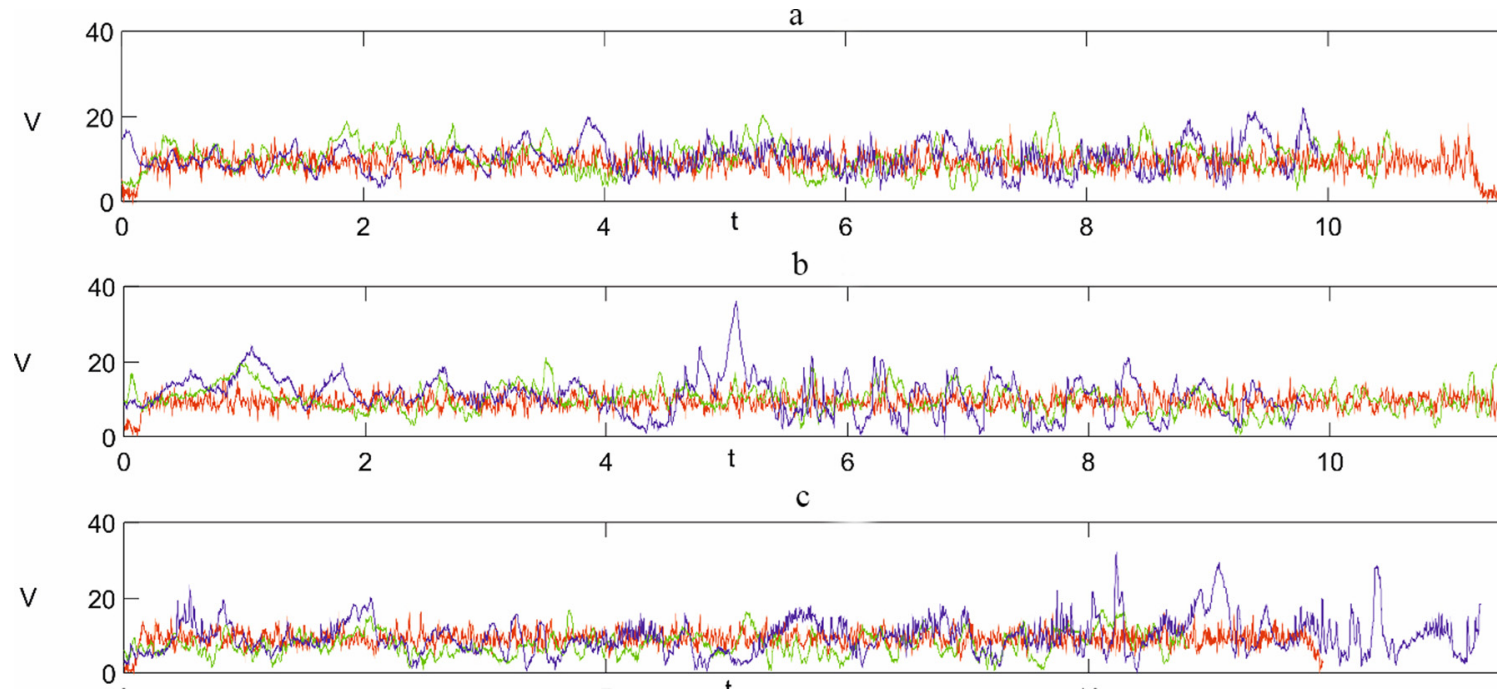

Fig. 6. Graphs of the value change of current cutting velocity $V_{t k}(\mathrm{~mm} / \mathrm{s})$ from the measurement time $t_{t k}(\mathrm{~s})$ of the coordinates of the position of the medical instrument.

scalloped and semilunar trajectories, the medical instrument tip passes a different path both relative to each other and with respect to the linear trajectory. Comparative graphs of the robot and the trained surgeon are shown in Figure 6. The graphs of the change of the value of current cutting velocity $V_{t k}(\mathrm{~mm} / \mathrm{s})$ from the measurement time $t_{t k}$ (s) of the coordinates of the position of the medical instrument for manual movements of the trained surgeon with a support point (color, blue), without support point (color, green) and when moving with the help of the robot (color, red) along the typical trajectories ( $\mathrm{a}$ - linear, b semilunar, c - scalloped). They reflect the advantages of a trained surgeon in comparison with untrained.

Trained working with the laser, the surgeon can maintain the value of the cutting velocity $V_{j}$ of one pass with manual movements close to the robot compared to the untrained surgeon. Also, the time for completion of measurements $t_{i}$ on each typical trajectory during manual movements of a trained surgeon is much closer to the robot. Table 1 shows the values of the cutting velocity $V_{j}$ of one pass $(j=1)$ along the linear, semilunar and scalloped trajectories for manual movements of both surgeons and for movements performed by the robot during the preliminary experiment.

Analyzing the values $V_{j}$ in Table 1 and the nature of the velocity graphs in Figures 5 and 6 , it is reasonable to assume the hypothesis that regardless of the type of trajectory, the robot stably maintains the necessary current cutting velocity $V_{j}$ of one pass compared with surgeons. For the confirmation of this hypothesis, it is expedient to conduct statistical experiments: every robotic trajectory must be passed by the robot and surgeon no less 
Table 1. The value of cutting velocity in one pass $V_{j}(j=1)$.

\begin{tabular}{llll}
\hline Trajectory & Linear & Semilunar & Scalloped \\
\hline Untrained surgeon with support point & 13603 & 187565 & 11854 \\
Trained surgeon with a support point & 15619 & 10948 & 9596 \\
Untrained surgeon without support point & 22933 & 19284 & 14443 \\
Trained surgeon without a support point & 11181 & 9979 & 7686 \\
Robot & 9127 & 9306 & 9192 \\
\hline
\end{tabular}
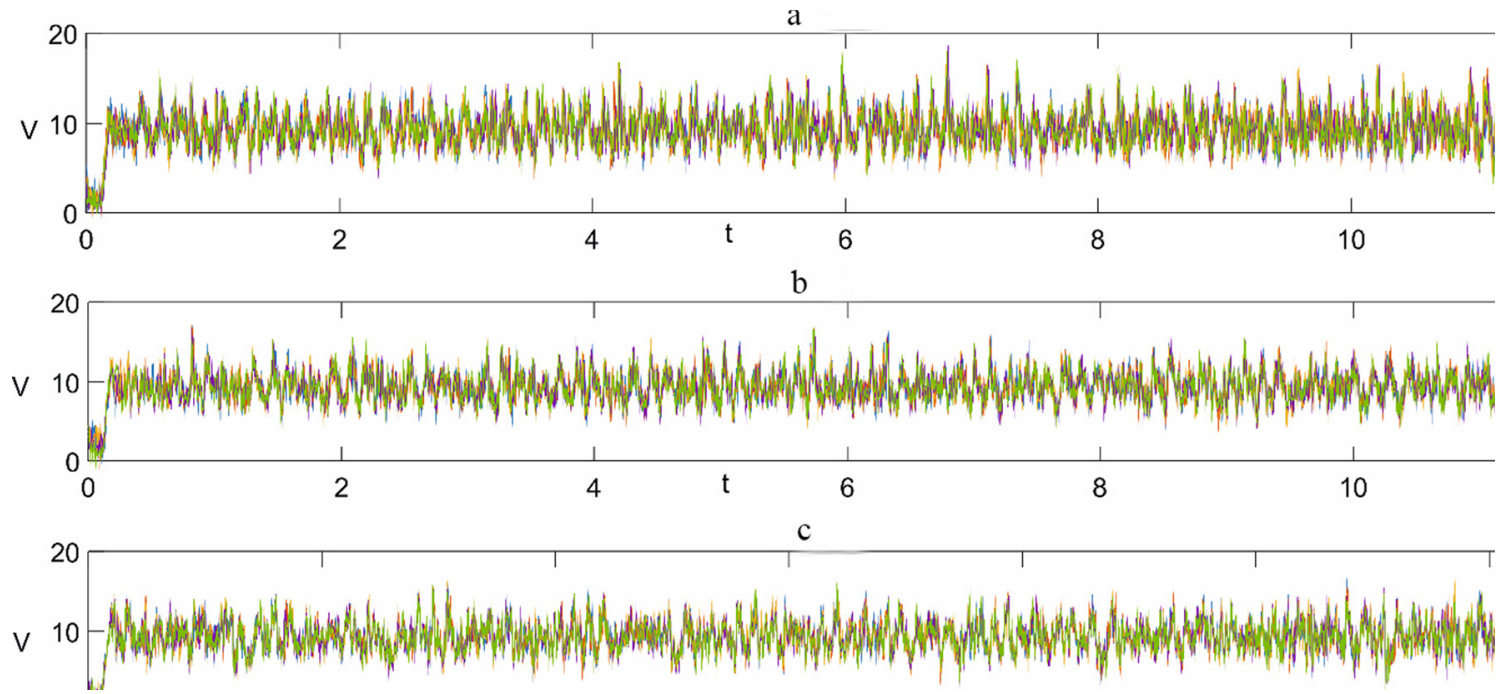

Fig. 7. Graphs of the change of the value of current cutting velocity $V_{t k}(\mathrm{~mm} / \mathrm{s})$ from the measurement time $t_{t k}(\mathrm{~s})$ of the coordinates of the position of the medical instrument moved by robot.

than five times $(j=5)$ and obtain the cutting velocity $V_{j}$ from each pass, determining the average cutting velocity $V_{m}$.

\subsection{The results of statistical experiments and the introduction of the new criterion}

In further experiments comparing the robot and the surgeon, only a surgeon trained using the diode laser participated. The results of statistical experiments when moving with the help of the robot along typical trajectories are shown in Figure 7 . The graphs of the change of the value of current cutting velocity $V_{t k}(\mathrm{~mm} / \mathrm{s})$ from the measurement time $t_{t k}(\mathrm{~s})$ of the coordinates of the position of the medical instrument for robot movements on typical trajectories with five passes each $(\mathrm{a}$ - linear, $\mathrm{b}$ - semilunar, c - scalloped). The results of statistical experiments for manual movements without a support point and with it are presented in Figures 8 and 9. Each passage for each typical trajectory on the graph is highlighted in its color. The graphs of the value change of current cutting velocity $V_{t k}$ $(\mathrm{mm} / \mathrm{s})$ from the measurement time $t_{t k}(\mathrm{~s})$ of the coordinates of the position of the medical instrument for manual movements without and with a support point accordingly on typical trajectories with 5 passes each (a linear, b - semilunar, c - scalloped).
Analyzing the results of statistical experiments, different time was established for completing the measurements $t_{i}$ on each typical trajectory during the manual movements of the surgeon. In contrast to manual movements when moving with the help of the robot, the end time of the measurement $t_{i}$ is the same. When determining the cutting velocity $V_{j}$ for each pass, the difference in manual movements with and without a support point is practically absent. When analyzing the obtained graphs in Figures $7-9$, it is also expedient to conclude about the identity of each trajectory regarding velocity in general, i.e. regardless of the choice of trajectory type, the nature of the dependence of the current velocity on the measurement time does not change. Therefore, from the data obtained, it is possible to determine the value of the mean velocity $V_{m}$ by combining all typical trajectories into one calculation. To calculate the average velocity by the standard ISO 9283: 1998, it is necessary to carry out atleast ten trajectories. When all typical trajectories are combined, the number of trajectories $j$ becomes 15 . Table 2 shows the values of the average cutting velocity $V_{m}$ and the cutting velocity accuracy of movement of the medical instrument $\delta_{V}$ according to formula (2) at a given cutting velocity $V_{n}=10 \mathrm{~mm} / \mathrm{s}$.

According to the data obtained, it turns out that the surgeon surpasses the robot's capabilities. However, this is when using the criterion of the cutting velocity accuracy of 

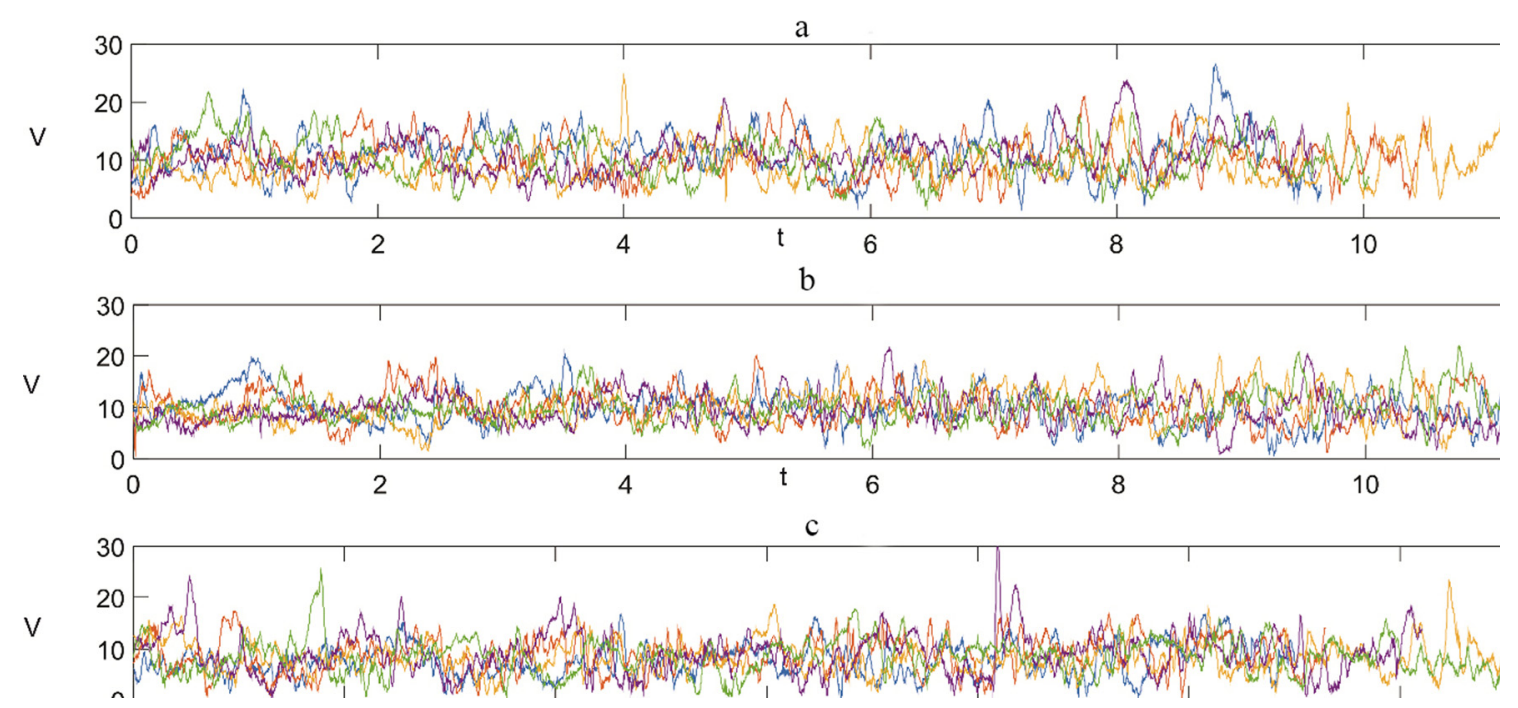

Fig. 8. Graphs of the change of the value of current cutting velocity $V_{t k}(\mathrm{~mm} / \mathrm{s})$ from the measurement time $t_{t k}(\mathrm{~s})$ of the coordinates of the position of the medical instrument (without support point on manual movement).

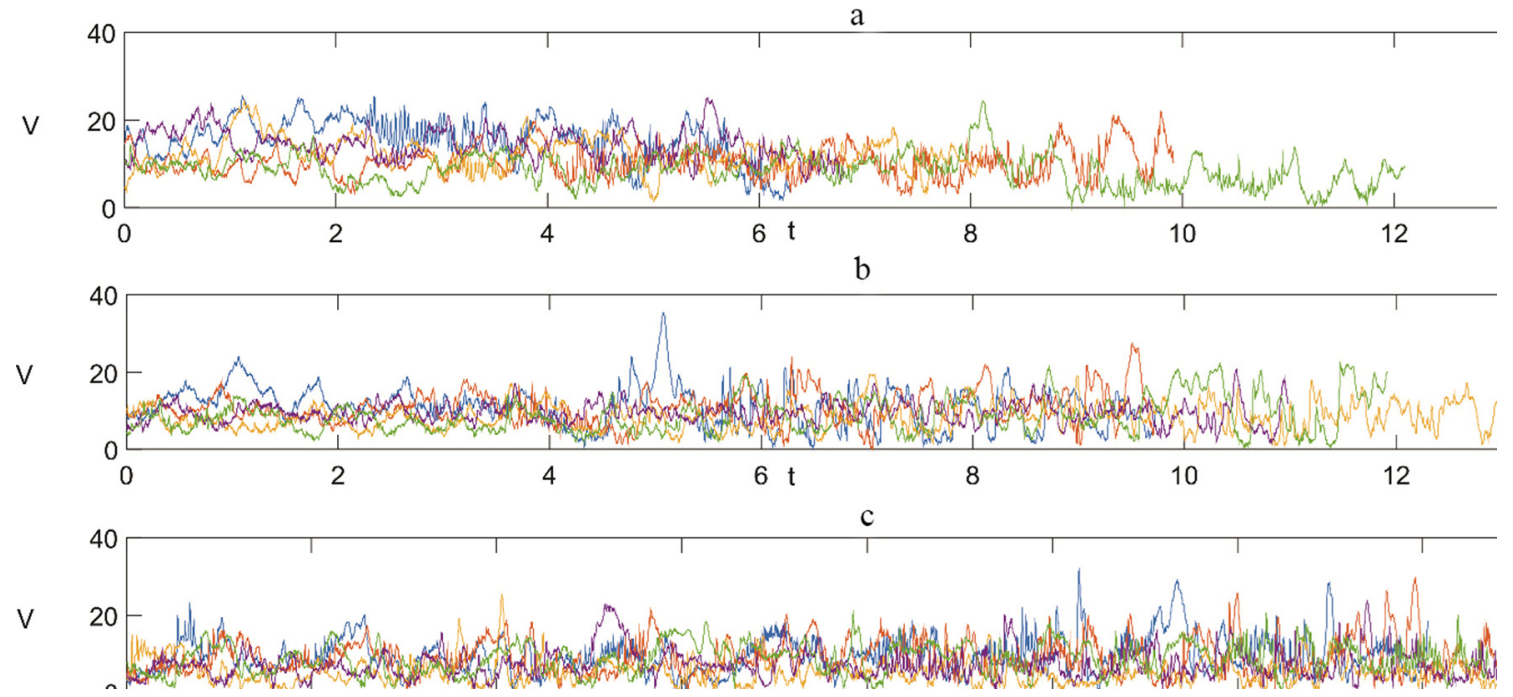

Fig. 9. Graphs of the change of the value of current cutting velocity $V_{t k}(\mathrm{~mm} / \mathrm{s})$ from the measurement time $t_{t k}(\mathrm{~s})$ of the coordinates of the position of the medical instrument (with support point on manual movement).

the medical instrument. The fact is that this is obtained only in a statistical experiment, where the average for several passages of manual movements was found. For example, the value $V_{j}$ of one of these passes is about $15 \mathrm{~mm} / \mathrm{s}$, in this case, when substituting it instead of $V_{m}$ in the formula for $\delta_{V}$, the velocity accuracy is $50 \%$, which is significantly higher than the value obtained for the robot. This criterion in the standard [30] is used to estimate the velocity accuracy of robots, so using this criterion to compare the manual movements and movements of the robot can come to an unfavorable result. Instead of this criterion for comparison of a surgeon and a robot, another, more feasible, is suggested: the arithmetic mean of the cutting velocity accuracy at each pass, which is calculated by the following formula:

$$
\delta_{V p r}=\frac{1}{Q} \cdot \sum_{j=1}^{Q}\left(\frac{\left|V_{\mathrm{j}}-V_{\mathrm{n}}\right|}{V_{\mathrm{n}}} \cdot 100 \%\right)
$$

The data on the cutting velocity accuracy $\delta_{V p r}$ are also presented in Table 2. According to the table, it is evident that with an unchanged value $\delta_{V}=\delta_{V p r}=6,8$ for the robot, $\delta_{V p r}$ increases significantly for manual movements. The proposed criterion $\delta_{V p r}$ takes better account of the internal spread of the values $V_{j}$ in comparison with $\delta_{V}$, which allows more accurate comparison of the movements of the surgeon and the robot. Due to the use of the arithmetic mean of the cutting velocity accuracy $\delta_{V p r}$, it is possible to obtain a more reliable comparison of the surgeon's manual movements and program movements of the robot. 
Table 2. The value of cutting velocity in one pass $V_{j}$ $(j=1)$.

\begin{tabular}{llll}
\hline Trajectory achieved & $V_{m}(\mathrm{~mm} / \mathrm{s})$ & $\delta_{V}(\%)$ & $\delta_{V p r}(\%)$ \\
\hline $\begin{array}{l}\text { Trained surgeon with } \\
\text { a support point }\end{array}$ & 9.9 & 0.5 & 19.2 \\
$\begin{array}{l}\text { Trained surgeon without } \\
\text { a support point }\end{array}$ & 9.6 & 3.7 & 7.7 \\
Robot & 9.3 & 6.8 & 6.8 \\
\hline
\end{tabular}

\section{Conclusions}

A new criterion $\delta_{V p r}$ is proposed, which, taking into account the internal spread of the cutting velocity $V_{j}$ of each passage, more accurately compares the manual movements and movements of the robot concerning $\delta_{V}$. Experimental researches of trajectories from program movements of the robot and manual movements of the surgeon were carried out. According to the proposed comparison criterion of the velocity of movement, it turned out that the robot is more accurate than the surgeon who conducts manual movements with a support point, and is more accurate in relation to the surgeon conducting manual movements without a support point, on average, by two times. The cutting velocity accuracy of the robot trajectory depends mainly on the setting of the drives and the resolution of the robot's position sensors. The cutting velocity accuracy of the robot can be reduced in two ways: by more precise adjustment of the drives or by choosing higher-quality components during the design of the robot.

It is possible to quantitatively confirm the hypothesis of stability of keeping the necessary cutting velocity $V_{j}$ of one pass of the robot, regardless of the type of trajectory in comparison with the surgeon, by quantitatively estimating the velocity deviation $V_{j}$ at each pass from the mean cutting velocity $V_{m}$. As it was mentioned above, the introduction of an additional criterion as velocity repeatability $\delta_{V r}$, needs further research.

Acknowledgements. The work was supported by the Ministry of Education and Science of Russia as part of State Assignment NO 9.3408.2017/4.6.

The work is carried out on the equipment of the Center of collective use of MSTU "STANKIN".

\section{References}

[1] A.A. Chunikhin, E.A. Bazikyan, A.A. Krasnovsky, N.V. Syrnikova, A.G. Chobanyan, Prospects of improvement of minimally invasive laser technologies in photodynamic therapy dental pathologies, Russ. Stomatol. 20 (2016) 228-231

[2] H. Jelinkova, Lasers for medical applications: diagnostics, therapy and surgery, Woodhead Publishing Limited, New Delhi, 2013

[3] L.A. Coldren, S.W. Coldren, M.L. Masanovic, Diode lasers and photonic integrated circuits, A John Wiley and Sons, New Jersey, 2012
[4] C.H. Chen, E.A. McCullagh, S.H. Pun, P.U. Mak, M.I. Vai, P.I. Mak, A. Klug, T.C. Lei, An integrated circuit for simultaneous extracellular electrophysiology recording and optogenetic neural manipulation, IEEE Trans. Biomed. Eng. 64 (2017) 557-568

[5] A.A. Chuniknin, E.A. Bazikyan, N.A. Pikhtin, A laser unit for photodynamic therapy and robot-assisted microsurgery in dentistry, Tech. Phys. Lett. 43 (2017) 507-510

[6] E. Azma, Na. Safavi, Diode laser application in soft tissue oral surgery, J. Lasers Med. Sci. 4 (2013) 206-211

[7] E.A. Bazikyan, A.A. Chunikhin, Prospects of improvement of minimally invasive laser technologies in photodynamic therapy dental pathologies, Russ. J. Dent. 20 (2016) 228-231

[8] M. Gambacciani, S. Palacios, Laser therapy for the restoration of vaginal function, Maturitas 99 (2017) 10-15

[9] R. Fathi, M.L. Pfeiffer, M. Tsoukas, Minimally invasive eyelid care in dermatology: medical, laser, and cosmetic therapies, Clin. Dermatol. 33 (2015) 207-216

[10] C. LaRosa, A. Chiaravalloti, S. Jinna, W. Berger, J. Finch, Laser treatment of medical skin disease in women, Int. J. Women's Dermatol. 3 (2017) 131-139

[11] D. Bosanquet, G. Roblin, Congenital Epulis: a case report and estimation of incidence, Int. J. Otolaryngol. 1 (2009) 1-4

[12] M. Laus, M.A. Conti, A. Croce, Giant fibrous Epulis: a case report of a benign mass of the oral cavity, Int. J. Otolaryngol. Head Neck Surg. 5 (2016) 228-232

[13] M. Moreno-Sanchez, R. Gonzalez-Garcia, D. GonzalezBallester, L. Ruiz-Laza, C. Moreno-Garcia, F. Monje, What is the role of elective neck dissection in patients with squamous cell carcinoma of the upper jaw? Cirugia Oral. Maxilofac. 39 (2017) 22-27

[14] A.A. Chunikhin, M.Y. Sahakyan, S.I. Gazhva, E.A. Bazikyan, Development of nanosecond laser module built in the robotic multifunctional surgical complex for minimally invasive therapy of maxillofacial area pathology and estimation of its effects on blood plasma, Sovrem. Tehnolog. Med. 8 (2016) 30-35

[15] R.M. Huertas, E.D. Luna-Bertos, J.R. Amos-Torrecillas, F. M. Leyva, C. Ruiz, O. Garcia-Martinez, Effect and clinical implications of the low-energy diode laser on bone cell proliferation, Biol. Res. Nurs. 16 (2014) 191-196

[16] M. Berot, J.-C. Auregan, L. Imbert, H. Magoariec, E. Budyn, F. Zadegan, D. Hannouche, M. Bensidhoum, T. Hoc, Mechanics of osteoporotic trabecular bone, Mech. Ind. 13 (2012) 373-380

[17] N. El Gharbi, A. Benzaoui, E.E. Khalil, R, Kameel, Analysis of indoor air quality in surgical operating rooms using experimental and numerical investigation, Mech. Ind. 13 (2012) 123-126

[18] J.C. LaMattina, J. Alvarez-Casas, I. Lu, J.M. Powell, S. Sultan, M.W. Phelan, R.N. Barth, Robotic-assisted singleport donor nephrectomy using the da Vinci single-site platform, J. Surg. Res. 222 (2018) 34-38

[19] A. Bryant, B. Wei, G. Veronesi, R. Cerfolio, Robotic surgery: techniques and results for resection of lung cancer, IASLC Thoracic Oncology (Second Edition), 2018, pp. 283-288

[20] A. Zihni, W.D. Gerull, J.A. Cavallo, T. Ge, S. Ray, J. Chiu, L.M. Brunt, M.M. Awad, Comparison of precision and speed in laparoscopic and robot-assisted surgical task performance, J. Surg. Res. 223 (2018) 29-33

[21] A. Shademan, R.S. Decker, J.D. Opfermann, S. Leonard, A. Krieger, P.C.W. Kim, Supervised autonomous robotic soft tissue surgery, Sci. Transl. Med. 8 (2016) 337 
[22] B. Jerbic, G. Nikolic, D. Chudy, M. Svaco, B. Sekoranja, Robotic application in neurosurgery using intelligent visual and haptic interaction, Int. J. Simul. Model. 14 (2015) 71-84

[23] M. Svaco, P. Koren, B. Jerbic, J. Vidakovic, B. Sekoranja, F. Suligoj, Validation of three KUKA Agilus robots for application in neurosurgery, Adv. Serv. Ind. Robot. 49 (2018) 996-1006

[24] T. Essomba, L. Nouaille, M.A. Laribi, C.A. Nelson, S. Zeghloul, G. Poisson, Spherical wrist dimensional synthesis adapted for tool-guidance medical robots, Mech. Ind. 15 (2014) 217-223

[25] R.C.O. Locke, R.V. Patel, Optimal remote center-of-motion location for robotics-assisted minimally-invasive surgery, in: IEEE International Conference on Robotics and Automation, Italy, Roman, 2007, pp. 1900-1905
[26] K. Henn, G.G. Gubaidullin, J. Bongartz, J. Wahrburg, H. Roth, M. Kunkel, A spectroscopic approach to monitor the cut processing in pulsed laser osteotomy, Lasers Med. Sci. 28 (2013) 87-92

[27] G. Kronreif, W. Ptacek, M. Kornfeld, M. Furst, Evaluation of robotic assistance in neurosurgical applications, J. Robot. Surg. 6 (2012) 33-39

[28] L. Balasubramani, D.A. Milliken, J.H. Shepherd, T.E.J. Ind, Differences in hand movements and task completion times between laparoscopic, robotically assisted, and open surgery: an in vitro study, J. Robot. Surg. 5 (2011) 137-140

[29] L. Zhao, A. Joubair, P. Bigras, I.A. Bonev, Metrological evaluation of a novel medical robot and its kinematic calibration, Int. J. Adv. Robot. Syst. 12 (2015) 126

[30] Manipulating Industrial robots - performance criteria and related test methods, ISO 9283: 1998

Cite this article as: A.A. Vorotnikov, D.D. Klimov, E.V. Romash, O.S. Bashevskaya, Y.V. Poduraev, E.A. Bazykyan, A.A. Chunihin, Cutting velocity accuracy as a criterion for comparing robot trajectories and manual movements for medical industry, Mechanics \& Industry 18, $712(2017)$ 